Revue des patrimoines

Le patrimoine militaire et la question urbaine

\title{
Réflexion sur la reconstruction d'un monument historique : l'avenir du Manège militaire de Québec
}

Marc Grignon et Ayse Orbay

\section{OpenEdition}

Journals

Édition électronique

URL : http://journals.openedition.org/insitu/293

DOI : 10.4000/insitu.293

ISSN : 1630-7305

Éditeur

Ministère de la culture

Référence électronique

Marc Grignon et Ayse Orbay, "Réflexion sur la reconstruction d'un monument historique : l'avenir du Manège militaire de Québec », In Situ [En ligne], 16 | 2011, mis en ligne le 27 juin 2011, consulté le 19 avril 2019. URL : http://journals.openedition.org/insitu/293; DOI : 10.4000/insitu.293

Ce document a été généré automatiquement le 19 avril 2019

\section{(c)}

In Situ Revues des patrimoines est mis à disposition selon les termes de la licence Creative Commons Attribution - Pas d'Utilisation Commerciale - Pas de Modification 4.0 International. 


\section{Réflexion sur la reconstruction d'un monument historique : l'avenir $\mathrm{du}$ Manège militaire de Québec}

Marc Grignon et Ayse Orbay

Figure 1

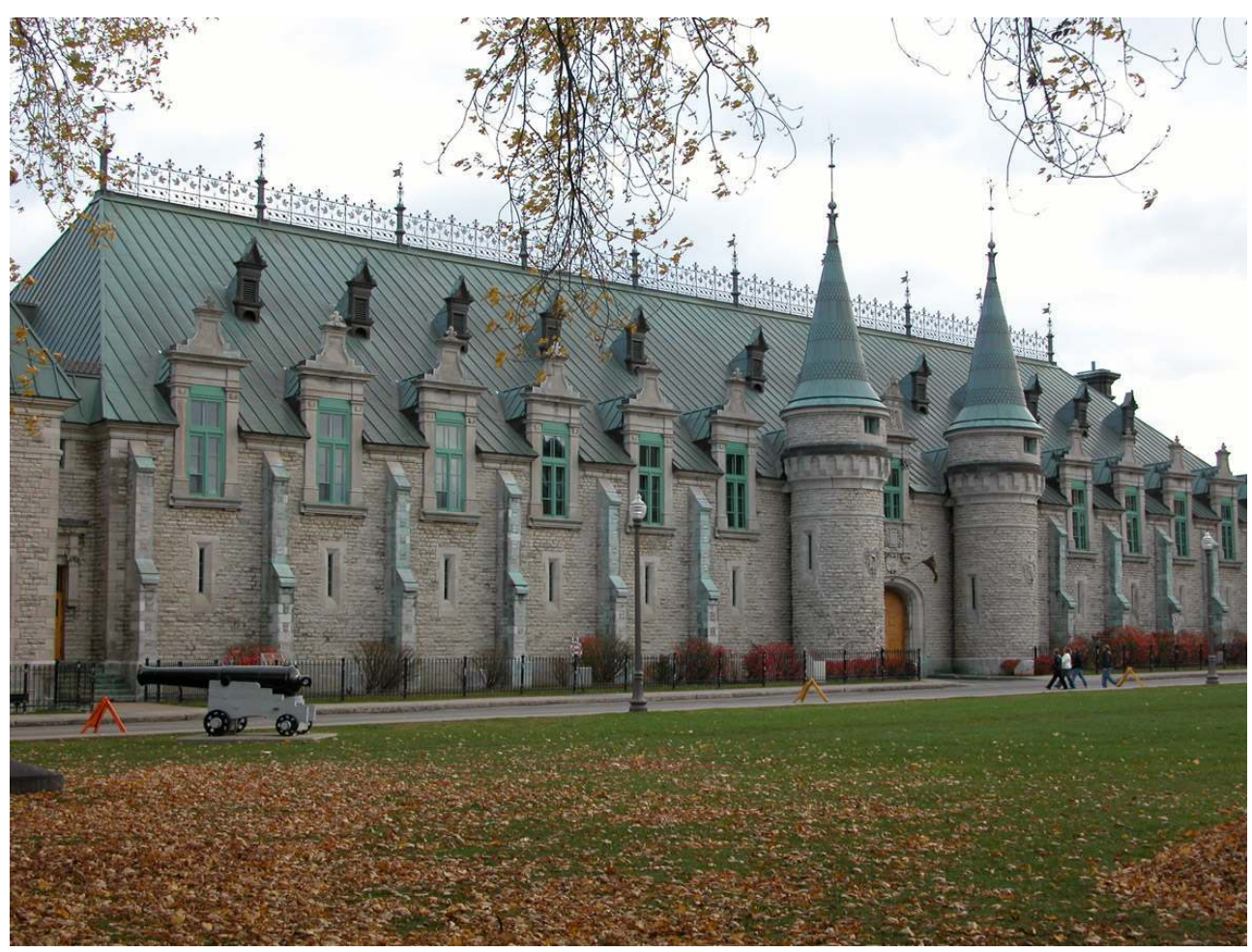

Manège militaire, Québec, par Eugène-Étienne Taché, 1887 : façade principale. Phot. MG, 2006. (c) MG. 
1 Le Manège ${ }^{1}$ militaire de la ville de Québec (fig. $\mathbf{n}^{\circ} \mathbf{1}$ ), construit en 1887 , comptait parmi les édifices les plus exceptionnels de cette ville ${ }^{2}$. Conçu par l'architecte Eugène-Étienne Taché (1836-1912), son vocabulaire se situait dans le sillage des portes Saint-Louis et Kent (1878-79) (fig. $\left.\mathbf{n}^{\circ} 2\right)$, tout en affichant des caractéristiques plus directement inspirées de la Renaissance française. Conçu pour servir de salle d'exercice pour les milices locales, c'était un des édifices les plus marquants de la fin du XIX ${ }^{\mathrm{e}}$ siècle à Québec, tenant bien sa place aux côtés de l'hôtel Le Château Frontenac, du Parlement et du Palais de Justice. C'est à travers ces bâtiments et quelques autres contemporains que s'est formée l'image actuelle de la ville de Québec, une ville qui vit avec son histoire, et qui a généralement été capable d'en assumer le poids tout en restant bien ancrée dans le présent.

Figure 2

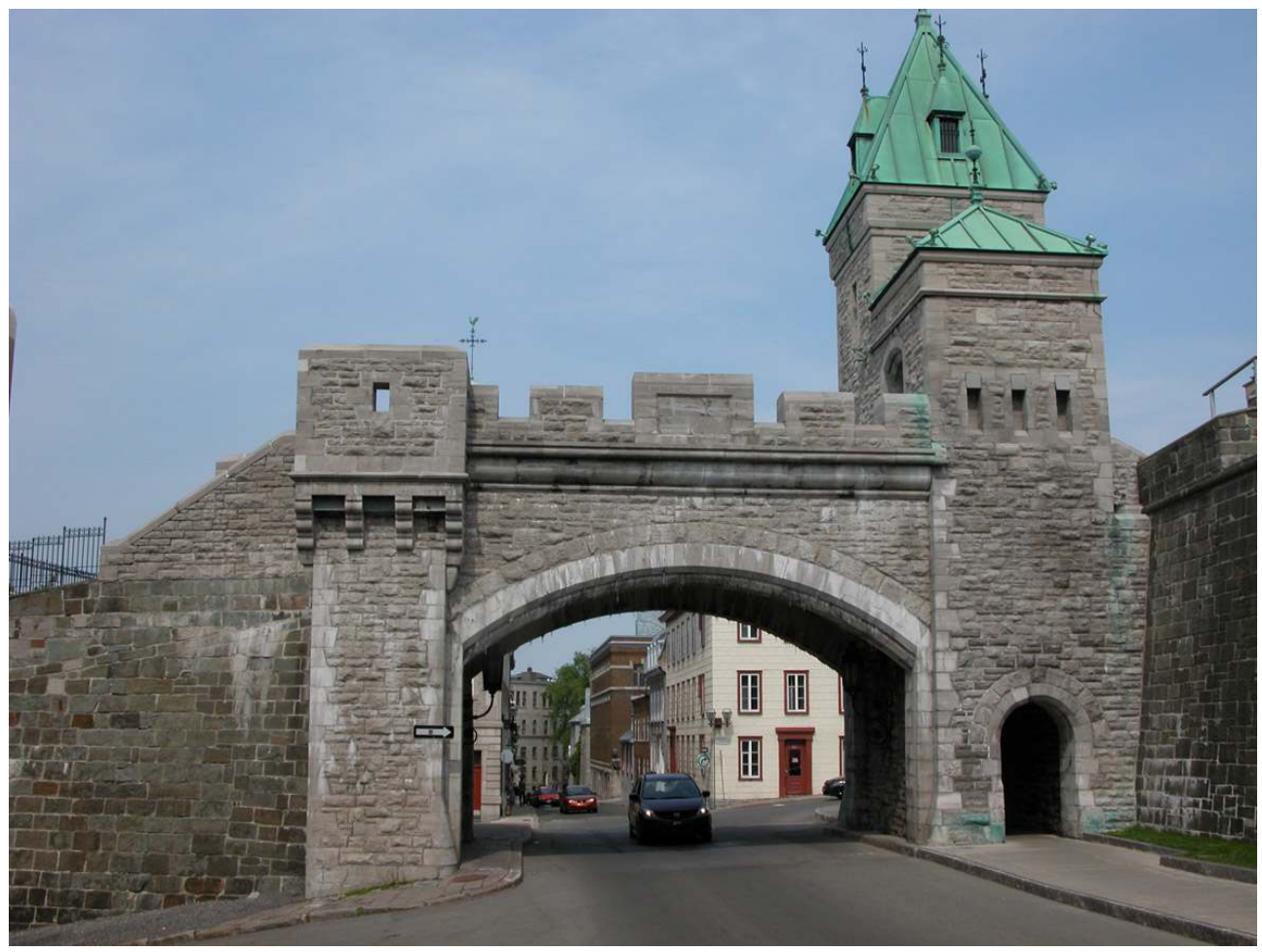

Porte Kent, Québec, par William Lynn et Charles Baillairgé. Phot. MG, 2007.

(C) MG.

2 Le Manège militaire a été détruit par un violent incendie dans la nuit du 4 au 5 avril 2008

(fig. $\mathbf{n}^{\circ}$ ), événement dont le caractère dramatique était amplifié par le début des célébrations entourant le quatrième centenaire de la fondation de la ville par Samuel de Champlain $^{3}$. Très rapidement, les propositions de reconstruction se sont multipliées, et même si l'intérêt de ces idées peut paraître très inégal aujourd'hui, l'ensemble révèle clairement l'intérêt que la population québécoise porte à ce bâtiment, son attachement envers un édifice emblématique de la ville. C'est dans ce contexte que le service des travaux publics canadiens - TPGSC, ou Travaux publics et Services gouvernementaux Canada - a lancé un processus de consultation publique sur une éventuelle reconstruction $\mathrm{du}$ monument, et a reçu les propositions et les commentaires du public entre le 12 mai et le 15 juin 2009. Depuis, Jean Baillargeon, expert-conseil responsable de la consultation publique, a examiné l'ensemble des commentaires et propositions et a publié son rapport 
en septembre $2009^{4}$. Ses conclusions ont été commentées et débattues dans les médias d'information, comme l'ont été les principaux concepts architecturaux qui avaient été présentés. Finalement, le 11 juin 2010, le gouvernement du Canada annonçait un budget d'environ 3,5 M (CAD) pour la reconstruction du bâtiment, avec des fonctions redéfinies pour loger des bureaux gouvernementaux, des espaces dédiés aux cérémonies des Forces militaires canadiennes, d'autres pour servir de musée militaire, en plus d'une «salle polyvalente » consacrée aux activités culturelles 5 . L'échéancier prévoyait le lancement de l'appel d'offres pour le concept architectural à l'automne 2010. Le nouveau concept doit être dévoilé au cours de l'été $2012^{6}$. Le moment est donc opportun de rappeler les grandes idées contenues dans le mémoire que nous avons déposé lors des consultations publiques de juin 2009. En effet, notre mémoire plaidait en faveur d'une réflexion approfondie sur le rôle de ce monument dans la ville de Québec avant qu'une orientation ne soit arrêtée. Il visait tout d'abord à proposer des principes à partir desquels un concept architectural pourrait être élaboré. Le texte qui suit reprend assez largement notre mémoire, ajoutant des précisions et des nuances là où elles nous semblaient nécessaires. En première partie, nous tenterons de faire le point sur les caractéristiques architecturales du bâtiment de Taché, démontrant que ses caractéristiques fondamentales ne se limitaient pas au style historiciste néo-Renaissance, précurseur du style Château, mais comprenaient en outre son organisation spatiale et son appartenance à une typologie architecturale précise. Dans le contexte d'un débat sur les principes d'une éventuelle reconstruction, une lecture purement stylistique est en effet trop réductrice, même si les caractéristiques stylistiques étaient les premières mentionnées comme justification du classement de l'édifice comme « lieu historique national $»^{7}$. Tout en respectant au mieux les paramètres définis lors de la consultation publique ${ }^{8}$, nous tentons de dégager les principes fondamentaux qui, à notre avis, devraient être suivis pour mettre en valeur les qualités les plus importantes du bâtiment original. Nous appuierons aussi l'idée - véhiculée dans les médias d'information depuis l'incendie - d'une salle multifonctionnelle pouvant servir au régiment des Voltigeurs, et pouvant aussi accueillir une variété d'événements qui, de par leur nature, nécessitent ce type d'espace : cérémonies officielles, expositions, etc. Nous proposons en réalité que le bâtiment devienne un point d'accueil pour les visiteurs du Parc des Champs de Bataille, avec sa grande salle meublée de chaises et de tables, ouverte aux promeneurs lorsqu'elle n'est pas requise pour d'autres fonctions. La seconde partie du texte expose quelques idées dessinées par l'architecte Ayşe Orbay. Il s'agit d'illustrer le potentiel des principes formulés dans la première partie, et de les compléter par l'image. En passant des principes aux formes architecturales, bien entendu, un saut important est effectué, et nous reconnaissons d'emblée que de nombreuses possibilités existent: les formes illustrées ne montrent évidemment pas les seules solutions compatibles avec les principes de la première partie. 


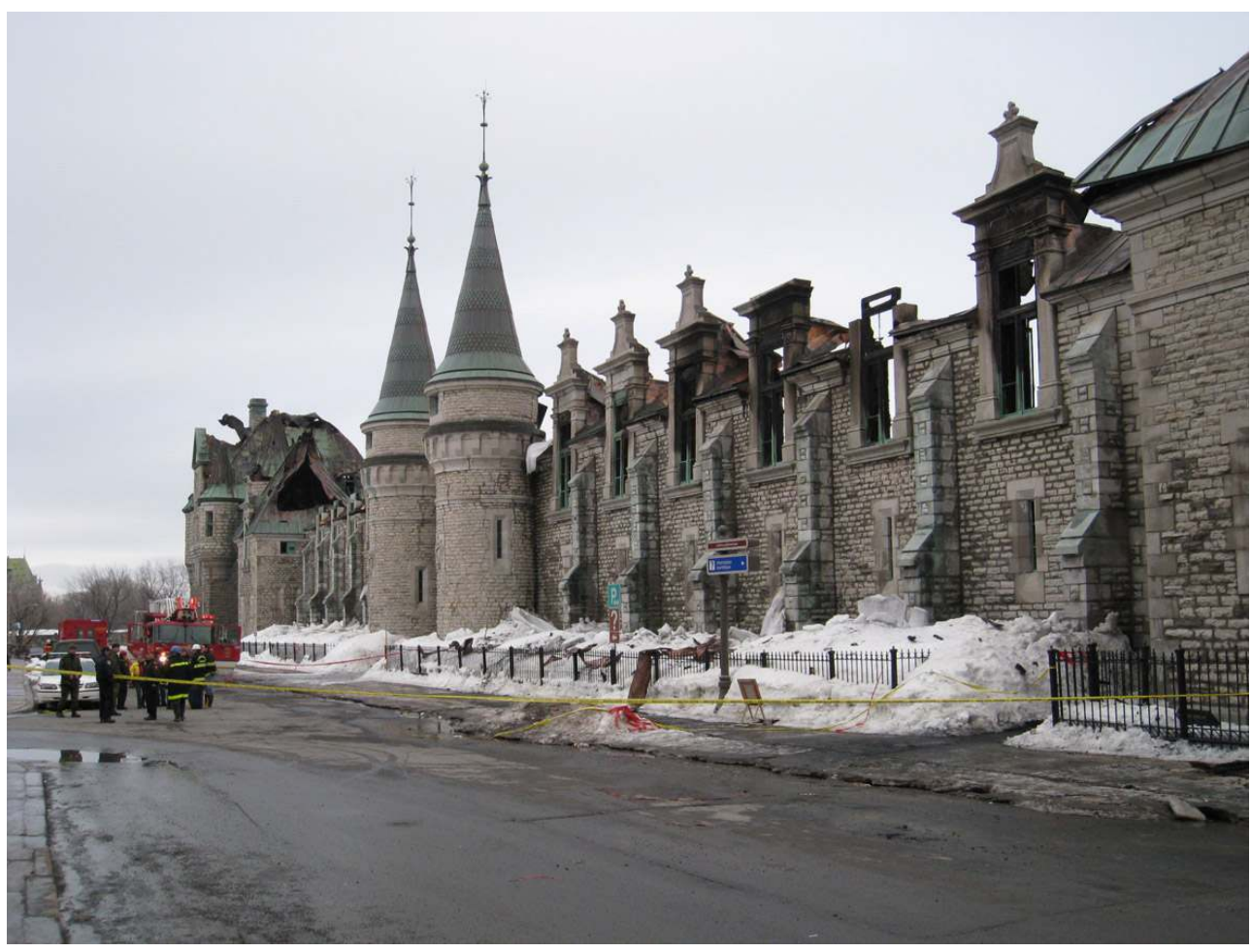

Vue du Manège militaire après l'incendie d'avril 2008 : façade principale. Phot. MG, 2008. (c) MG.

\section{Caractéristiques architecturales}

3 Le style architectural que Taché a choisi pour le Manège militaire est inspiré de la Renaissance française. En même temps, l'interprétation que l'architecte a faite des modèles historiques est très personnelle, notamment dans le rapport entre l'extérieur et l'intérieur, et pour bien comprendre son bâtiment, il est nécessaire d'en considérer les qualités architecturales d'une manière plus complète. C'est ainsi qu'il deviendra possible de mieux mettre en valeur les éléments originaux ayant été conservés. En outre, sur la base d'une telle analyse, on pourra exploiter et valoriser certaines caractéristiques fondamentales du bâtiment et choisir les détails disparus devant être reproduits. Dans cette perspective, il est nécessaire que le projet de reconstruction du Manège militaire s'appuie clairement sur les principes architecturaux du bâtiment de Taché, même dans les parties qui seront traitées de manière explicitement contemporaine. Et c'est à lumière de tels principes que les projets de reconstruction devraient être évalués. Pour nous, l'idée essentielle reste de faire en sorte que le projet de reconstruction du Manège aboutisse à une œuvre authentique, cohérente dans une perspective actuelle, et respectueuse d'un passé complexe qui nous est parvenu de manière fragmentaire.

\section{Éléments stylistiques néo-Renaissance}

4 À la Renaissance, le château français conserve plusieurs éléments de fortification empruntés au Moyen Âge, bien que ces éléments soient la plupart du temps dépourvus de 
fonction défensive réelle. Ce sont des souvenirs d'une aristocratie guerrière et de ses valeurs chevaleresques. Les fossés, les tours rondes, le donjon font partie de ces éléments, mais ils sont agencés dans une composition plus formelle, généralement symétrique, comme c'est le cas aux châteaux de Chambord ou de Chenonceau.

On peut voir plusieurs exemples de cette "manière moderne» dans les recueils de Jacques Androuet du Cerceau ${ }^{9}$, certaines planches montrant des bâtiments très comparables au Manège militaire de Taché. La forme générale du Manège militaire - avec son grand volume horizontal, plutôt allongé, coiffé d'une haute toiture et ponctué de lucarnes maçonnées - est empruntée assez directement à cette architecture renaissante. Les tours en poivrière disposées symétriquement de part et d'autre de l'entrée principale appartiennent au même répertoire formel, tout comme les hautes souches de cheminées (fig. $\left.\mathbf{n}^{\circ} \mathbf{4}\right)$. En partie pour la protection des toitures et en partie pour l'animation qu'elles produisent dans la silhouette des bâtiments, ces hautes cheminées se retrouvent dans de nombreux châteaux français, grands et petits. Elles sont bien entendu à l'honneur à l'époque de François $1^{\mathrm{er}}$, au château de Chambord par exemple. Elles complétaient la silhouette du Manège militaire en renforçant aux extrémités la symétrie de la composition. Restés debout au lendemain de l'incendie, ces éléments de maçonnerie devraient être rétablis.

\section{Figure 4}

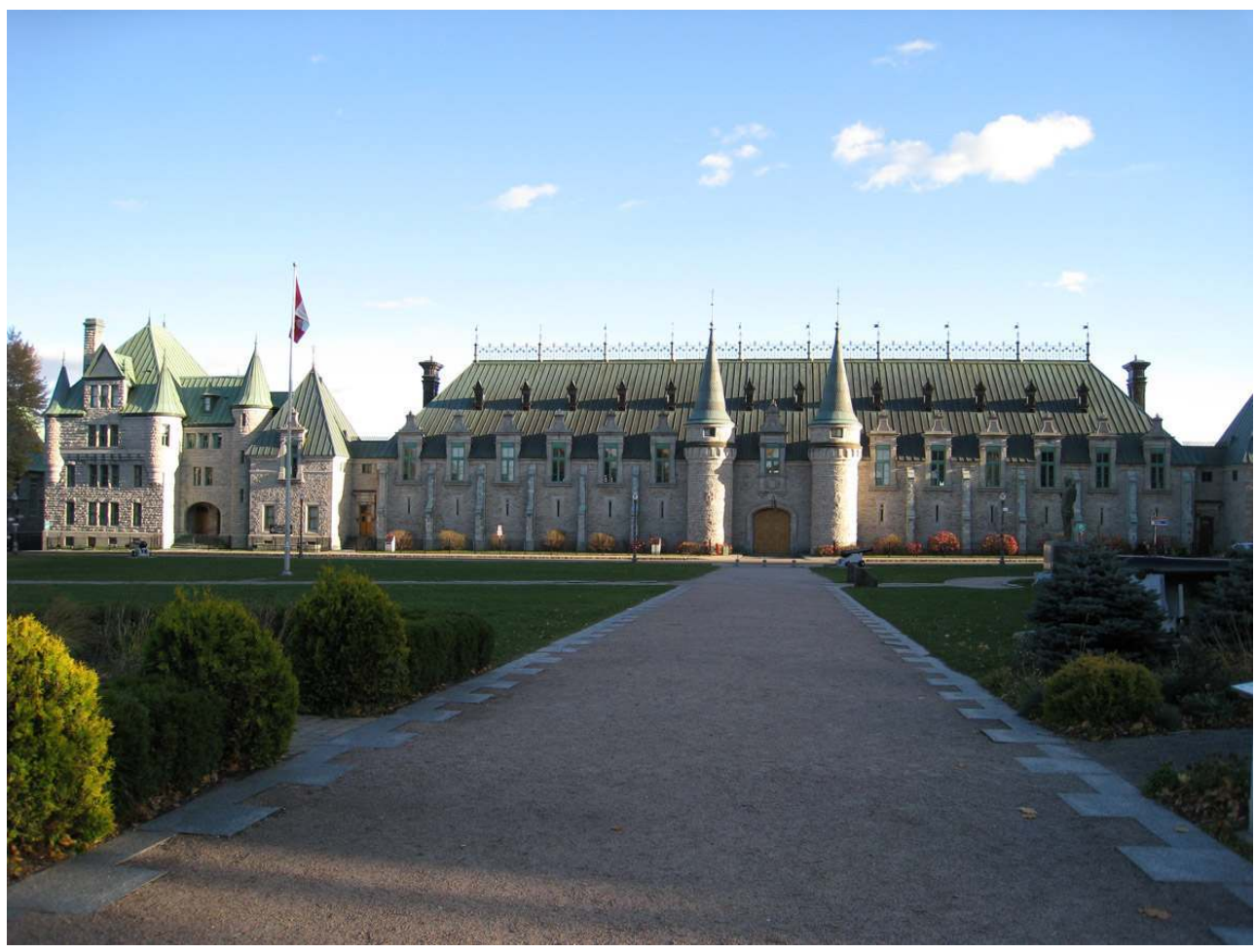

Manège militaire, Québec, par Eugène-Étienne Taché, 1887 : vue générale. Phot. MG, 2007. (c) MG.

\section{Volumes et toitures}

Les hautes toitures du Manège militaire, comme dans l'architecture de la Renaissance, soulignaient l'individualité des volumes (fig. $\mathbf{n}^{\circ} 5$ ). En effet, dans un château français de la 
Renaissance, chaque corps de bâtiment, chaque tour, chaque tourelle d'escalier possède sa propre toiture, et les différents volumes sont fortement individualisés, intégrés dans une composition où l'idée d'agencement de parties distinctes est une caractéristique essentielle. Ce principe de composition se retrouvait au Manège militaire, dont la toiture principale répondait aux toits coniques des tours en façade, aux toits pyramidaux des pavillons aux extrémités et à la toiture en appentis sur le côté sud. La forme générale de la toiture principale, avec sa terminaison en croupes, exprimait clairement la volumétrie du corps principal, et cette forme était tout à fait conséquente de l'organisation spatiale à l'intérieur. La toiture principale du nouveau bâtiment, même si sa forme était différente de la toiture originale, devrait contribuer à rétablir la cohérence de l'ensemble de la composition, et les autres toits devraient être réparés ou rétablis.

Figure 5

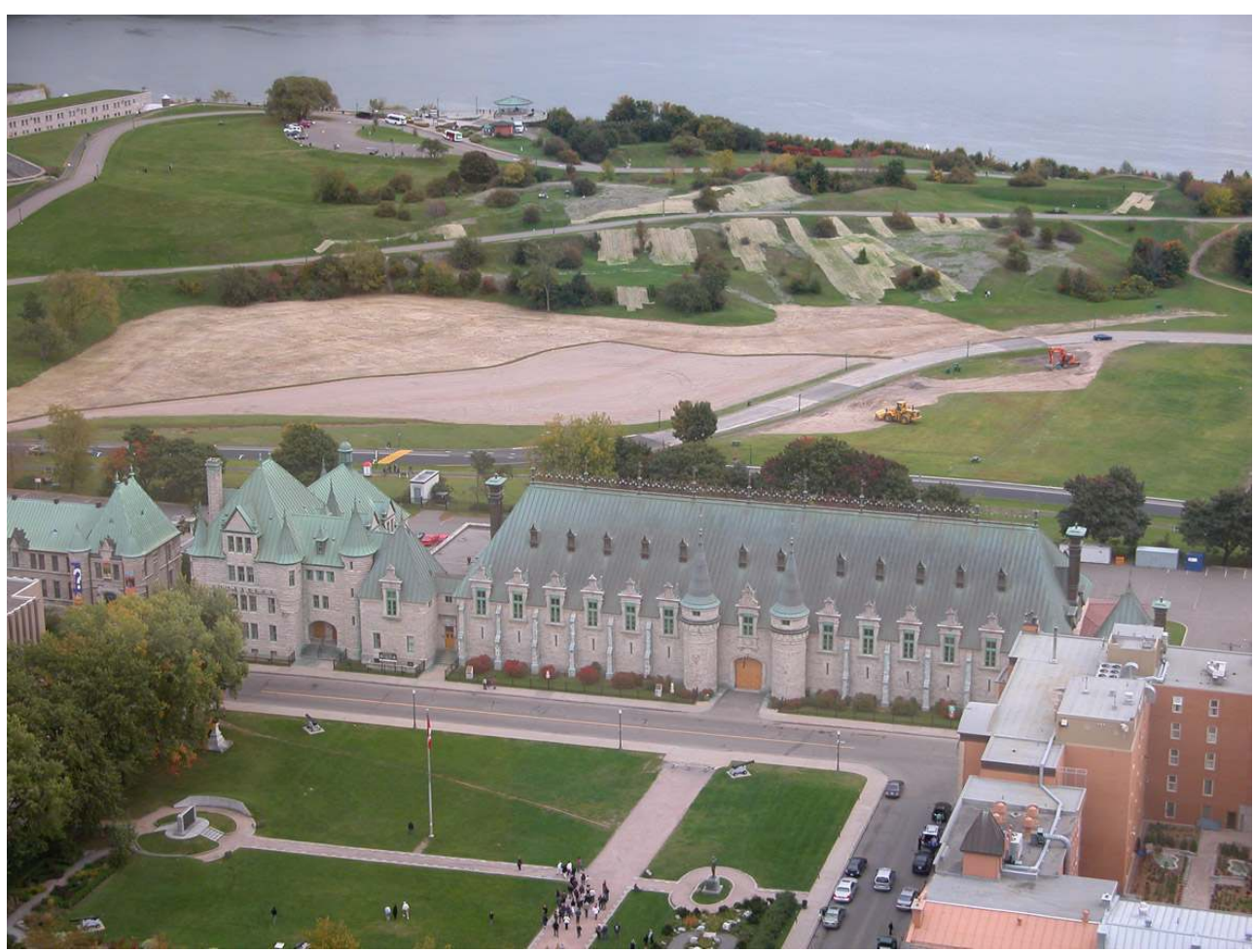

Manège militaire, Québec, par Eugène-Étienne Taché, 1887 : vue aérienne. Phot. MG, 2007.

(c) MG.

\section{Matériaux extérieurs et cohérence visuelle}

7 La cohérence architecturale du Manège militaire à l'extérieur dépend directement du rapport entre la forme des différentes toitures, toutes dans un même matériau (cuivre), et de leur contraste avec l'appareil de maçonnerie (fig. $\left.\mathbf{n}^{\circ} \mathbf{6}\right)$. La simplicité de ce contraste entre les murs de pierre et les toitures de cuivre devrait rester un principe de base dans la reconstruction. Il faudrait faire très attention à ne pas trahir cette qualité si de nouveaux matériaux étaient employés. 


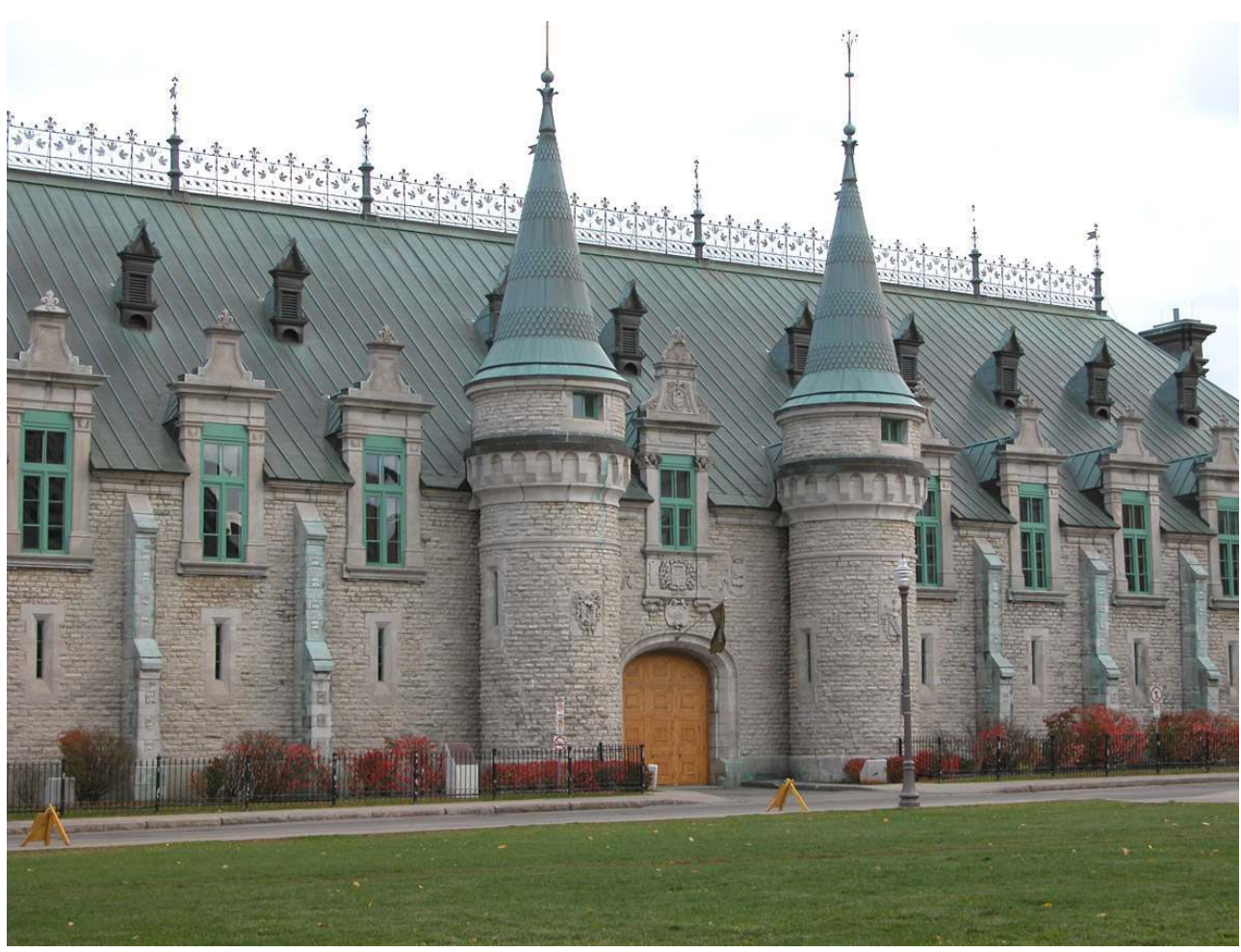

Manège militaire, Québec, par Eugène-Étienne Taché, 1887 : façade principale. Phot. MG, 2006. (c) MG.

\section{Répétition et sérialité}

La composition de l'ensemble possède aussi un caractère très animé, associé aux séries d'éléments répétitifs comme les lucarnes, les contreforts, les bandes de cuivre de la toiture, l'ornement du faîtage, etc. Taché a exploité pleinement cette sérialité - dérivée de la structure par travées - pour susciter la tension visuelle propre au bâtiment. Dans l'architecture renaissante, on remarque la même volonté d'employer à des fins décoratives cette sérialité structurale qui dérive assez directement du gothique, bien différente des surfaces lisses qu'on verra plus tard. Parmi les éléments répétitifs du Manège militaire, les lucarnes passantes sont sans aucun doute celui qui attire le plus l'attention. $\mathrm{Au} \mathrm{XVI}{ }^{\mathrm{e}}$ siècle, ces lucarnes sont généralement construites en maçonnerie et semblent prolonger des portions de mur au niveau des toitures. En brisant la ligne de rencontre entre les murs et les toits, ces lucarnes participent fortement à l'effet "pittoresque» de l'architecture de cette période. On les retrouvait, par exemple, au Palais des Tuileries (1564-72, détruit) à Paris.

Plusieurs lucarnes du Manège militaire étaient encore debout au lendemain de l'incendie, bien que certaines aient été fortement endommagées (fig. $\mathbf{n}^{\circ} 7$ ). Comme on le verra plus loin, elles constituaient un élément clé de l'interprétation que Taché a faite de l'architecture française. Il suffit ici de souligner que ces lucarnes participaient directement au caractère sériel de la composition extérieure et que la plupart ont survécu à l'incendie, même si elles ont été abîmées ; par conséquent, il est essentiel de les rétablir. 
D’une façon plus générale, le principe de la sérialité des éléments architecturaux constitue la base de la composition extérieure.

Figure 7

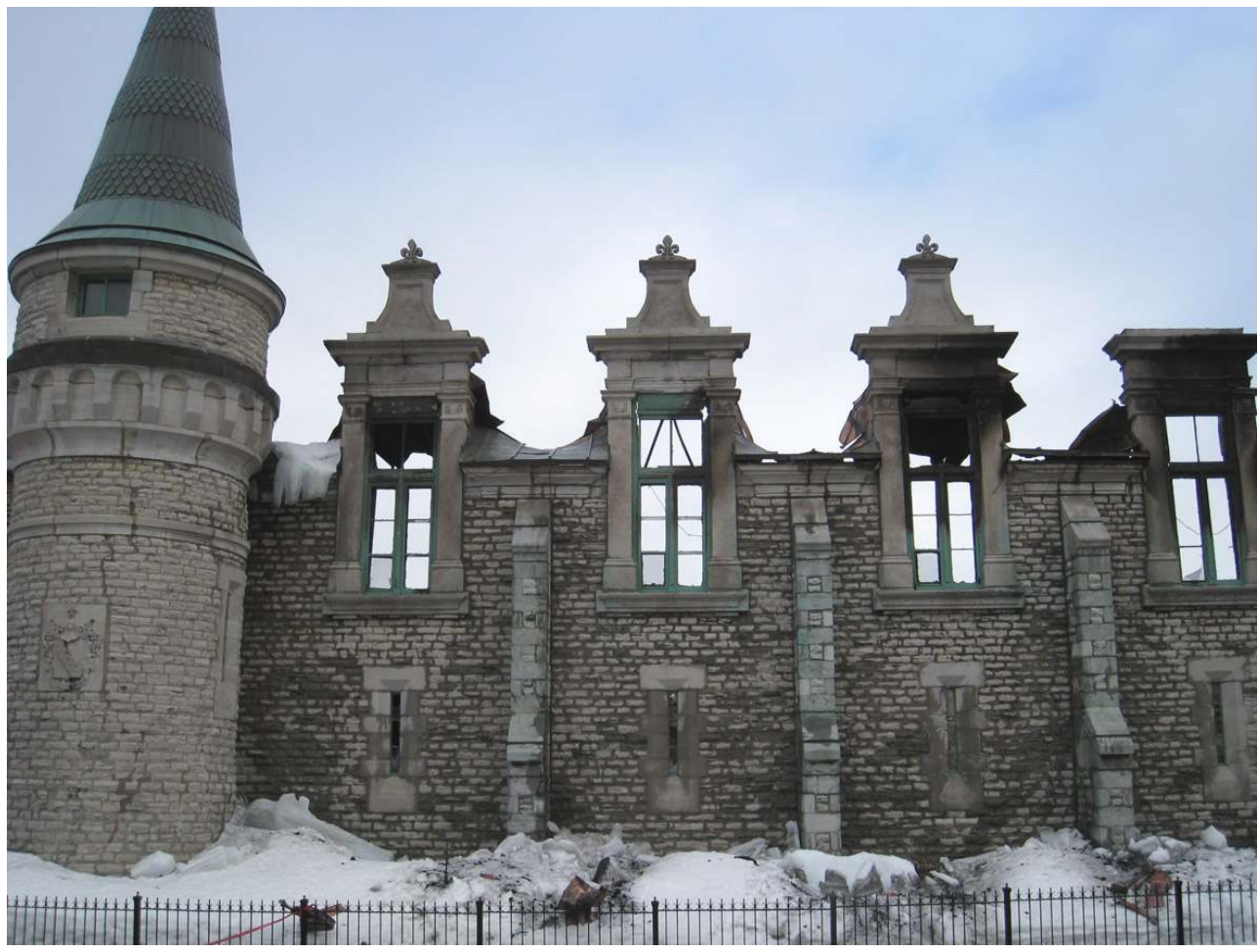

Vue du manège militaire après l'incendie d'avril 2008 : détail de la façade principale. Phot. MG, 2008. (c) MG.

\section{L'espace intérieur et l'organisation du bâtiment en coupe}

Au-delà de leur participation à l'effet pittoresque du bâtiment, les lucarnes passantes ont une fonction architecturale spécifique dans l'architecture de la Renaissance. Elles servent à éclairer l'étage appelé le surcroît, espace hybride partiellement contenu dans la maçonnerie et partiellement situé sous les combles (le plancher supérieur étant disposé en dessous des lucarnes, donc plus bas que la tête des murs).

11 Au Manège militaire, Taché a pris une grande liberté avec cette organisation du bâtiment en coupe : il a éliminé le plancher du surcroît pour le remplacer par la mezzanine qui fait le tour de la salle d'exercice. Cette décision consiste ni plus ni moins à évider l'intérieur du bâtiment, tout en conservant une trace de la disposition historique. Il a ensuite installé un grand plafond en bois exactement au niveau de la tête des murs, coupant ainsi les lucarnes en deux parties : la partie inférieure éclaire la salle principale, alors que la partie supérieure sert à illuminer l'intérieur du toit.

12 Tout l'intérieur du Manège militaire ayant été détruit, peu d'éléments peuvent être conservés en tant que tels. Compte tenu de cette situation, les idées de Taché devraient être mises au service des besoins actuels. Dans une perspective architecturale, une plus grande ampleur pourrait être donnée à la salle et un meilleur parti pourrait être tiré de ces lucarnes passantes. En effet, même si une reconstitution intégrale serait à notre avis très malvenue, il faut aussi éviter la création d'une structure intérieure étrangère à 
l'édifice original: une appropriation contemporaine de cet espace intérieur devrait se faire de manière à valoriser les qualités architecturales des éléments conservés.

Étant donné l'intérêt et l'originalité du geste de Taché, une structure en mezzanine à la même hauteur que la première devrait être mise en place. La présence d'une mezzanine à cette hauteur donne en effet tout son sens à la fenestration du bâtiment et exprime le lien que Taché a voulu établir avec les sources françaises. Mais cette nouvelle mezzanine pourrait être dimensionnée et aménagée selon les besoins actuels; elle pourrait être plus ou moins profonde, comprendre des avancées ou des reculs, etc. Du point de vue fonctionnel, une mezzanine agrandie serait un très bon endroit pour installer des vitrines à la disposition du Musée des Voltigeurs.

14 Le même principe vaut pour la charpente de la toiture et le plafond: ces éléments entièrement détruits devraient être conçus d'une manière contemporaine et bien mettre en valeur les caractéristiques architecturales des éléments conservés (au lieu de reproduire les éléments disparus, ou de verser dans la nouveauté arbitraire). Plus précisément, nous pensons ici aux qualités spatiales de l'architecture intérieure, car il y a maintenant une occasion intéressante de clarifier le rapport avec l'extérieur du bâtiment. La nouvelle charpente pourrait très bien être laissée entièrement ouverte. Un nouveau plafond, s'il était nécessaire, pourrait être situé à une hauteur différente. En réalité, du point de vue architectural, il serait logique et intéressant d'exprimer complètement les grandes lucarnes dans l'espace principal. Si les concepteurs d'un nouveau projet jugent nécessaire de fermer l'espace intérieur d'un plafond, celui-ci ne devrait pas être une reconstitution de celui qui a été détruit. Précisons que la liberté d'interprétation suggérée ici s'appuie sur le fait que les éléments originaux sont complètement détruits, et elle ne se justifiera que dans la mesure où le projet contribuera à mettre en valeur les éléments conservés par une interprétation témoignant d'une véritable compréhension de l'architecture originale.

\section{Typologie}

15 Par sa grande salle d'exercice correspondant au volume principal de l'édifice, le Manège militaire appartient aussi à un type architectural répandu en Amérique du Nord pendant la seconde moitié du XIX siècle. Identifiés en anglais comme drill halls ou armouries, ces manèges militaires constituaient les bases des milices locales, et comprenaient principalement une grande salle d'exercice accompagnée d'un dépôt d'armes ${ }^{10}$. La plupart de ces bâtiments empruntaient un style d'inspiration médiévale: néo-roman, néogothique, néo-Tudor, etc. Ayant perdu leur fonction au XX siècle, un grand nombre de ces manèges militaires a disparu.

$16 \mathrm{Au} \mathrm{XX}^{\mathrm{e}}$ siècle, les armouries et drill halls conservés ont souvent été utilisés pour tenir des expositions temporaires. La plus célèbre est évidemment l'Armory Show de 1913 à New York, exposition d'artistes modernes pour laquelle les organisateurs avaient loué le 69th Regimental Headquarters de la New York National Guard. À Québec, c'est l'Exposition provinciale qui a été présentée au Manège militaire pendant quelques années, dès 1887. Ces événements ont l'intérêt de montrer comment les caractéristiques fondamentales de ces bâtiments peuvent répondre à un besoin de salles d'expositions temporaires. 


\section{Une nouvelle fonction : Accueil des Plaines d'Abraham}

17 Par sa position sur la rue Saint-Louis - axe principal d'entrée dans le Vieux-Québec - en bordure des Plaines d'Abraham, le Manège militaire se prêterait très bien à l'accueil des promeneurs utilisant le parc, été comme hiver (voir fig. $n^{\circ} 5$ ). La grande salle, un peu comme le Chalet du Mont-Royal à Montréal ${ }^{11}$, pourrait servir de salle de repos, avec tables et chaises, où les promeneurs pourraient s'asseoir, se restaurer avec la nourriture qu'ils auront apportée ou achetée à un buffet (fig. n8). Par ailleurs, cette salle multifonctionnelle pourrait accueillir toutes sortes d'événements spéciaux dont la nature demande un espace ouvert de cette dimension (réceptions officielles, cérémonies, expositions, etc.).

Figure 8

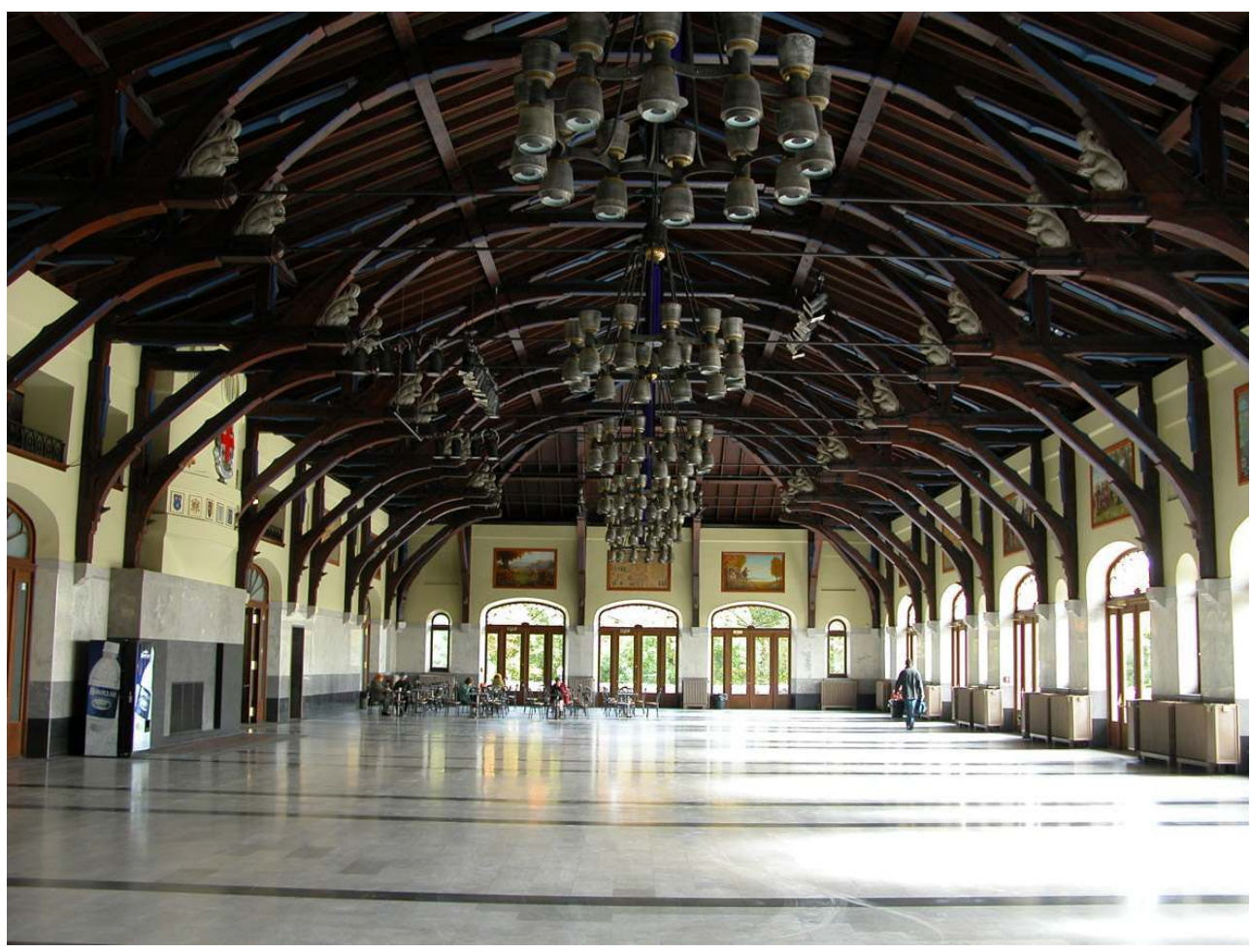

Chalet du Mont-Royal, Montréal, par Aristide Beaugrand-Champagne, 1931-32. Phot. MG, 2004. (c) MG.

\section{L'annexe en forme d'appentis}

Utilisé comme bâtiment d'accueil desservant les Plaines d'Abraham, le Manège militaire devrait être facilement accessible du côté du parc. En effet, le côté sud du manège ne devrait pas être traité comme l'arrière d'un bâtiment n'ayant qu'une seule façade. Au contraire, le caractère tridimensionnel de l'architecture de Taché devrait être mis en valeur. Dans cette perspective, la partie en appentis qui enveloppait le volume principal, complètement détruite (fig. $\mathbf{n}^{\circ} \mathbf{9}$ ), pourrait être réinterprétée de manière à donner plus d'ouverture sur le parc. Dans l'édifice original, la toiture en appentis prenait appui sur le volume principal juste en dessous des lucarnes passantes, et elle venait par conséquent 
souligner le niveau de la mezzanine à l'intérieur - jouant sur l'idée française du surcroît. Cependant, l'appentis dissimulait aussi la partie inférieure du volume principal, pourtant traité comme un extérieur (avec les mêmes contreforts que ceux qui rythmaient la façade nord, par exemple). Il semble y avoir là une contradiction, mais, grâce aux plans originaux, nous savons que Taché avait prévu d'interrompre cet appentis dans la partie centrale, entre les deux tours d'escalier ${ }^{12}$. De cette manière, il laissait visible le centre de la façade arrière, avec sa grande porte en anse de panier qui répondait directement à celle de la façade principale.

Figure 9

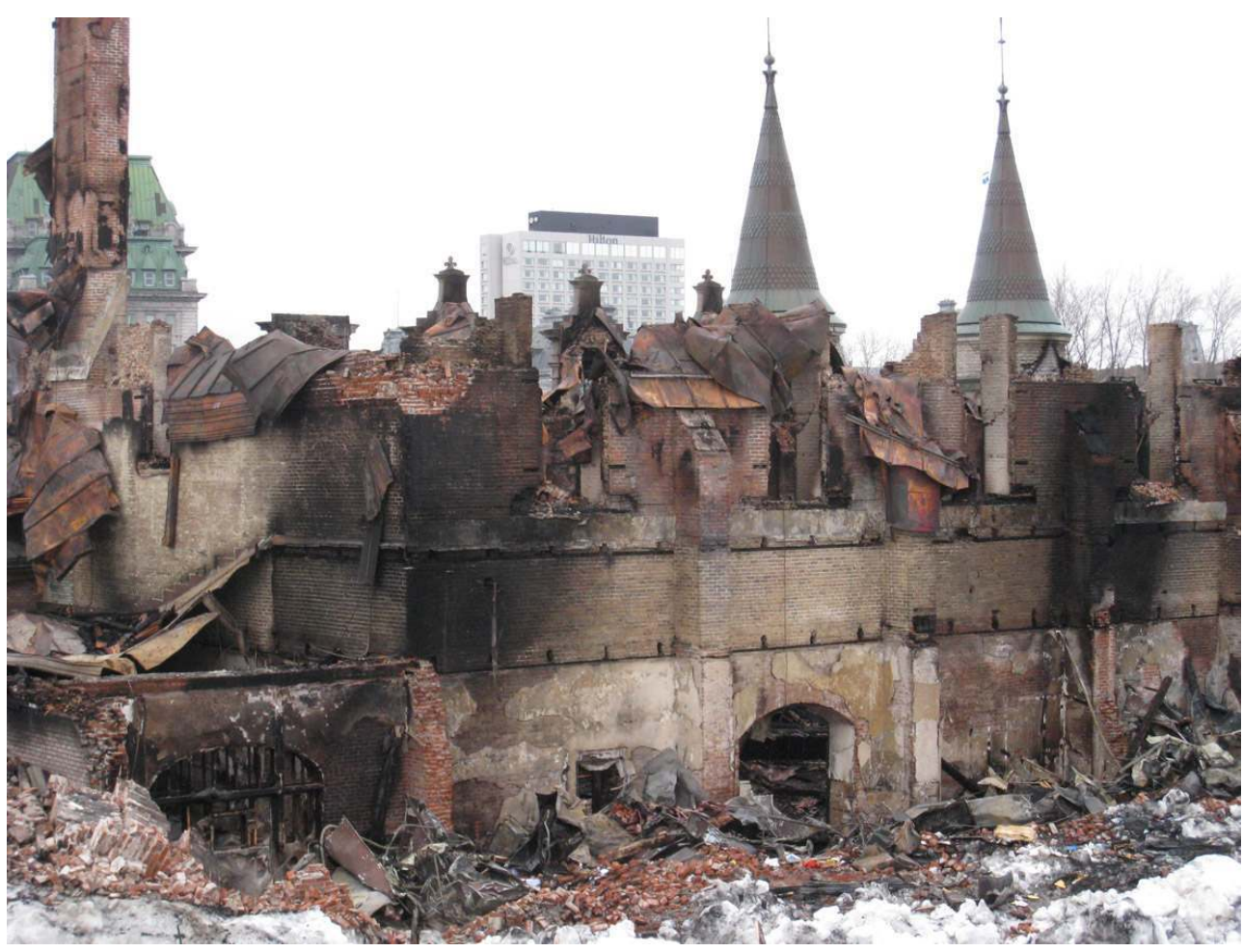

Vue du manège militaire après l'incendie d'avril 2008 : façade sud, avec l'empreinte de l'appentis. Phot. MG, 2008.

(c) MG.

Toute cette section pourrait être réinterprétée avec une liberté relative : son échelle intermédiaire est intéressante pour développer un lien visuel et spatial avec le parc, tout en appuyant la monumentalité du volume principal. Afin de conserver l'autonomie fonctionnelle de la salle principale, c'est elle qui devrait contenir les principaux services d'accueil : café, comptoir de documentation, toilettes publiques. Des stratégies formelles contemporaines pourraient ici être employées pour bien mettre en valeur les murs conservés du corps de bâtiment principal.

\section{Extérieur et aménagement paysager}

Une conséquence importante de l'utilisation du Manège militaire comme bâtiment d'accueil sur les Plaines d'Abraham serait la nécessité d'un nouvel aménagement paysager, davantage tourné vers le parc. En particulier, l'espace de stationnement qui 
ceinture le bâtiment nous paraît fondamentalement incompatible avec cette fonction d'accueil. Un rapport direct et fluide entre le parc et l'édifice devrait être favorisé.

Par ailleurs, le terrain de parade devant la façade principale du bâtiment devrait conserver son aménagement actuel. Tout au plus, des corrections mineures pourraient lui être apportées.

Pour conclure cette partie, il nous paraît évident qu'une recherche historique est nécessaire pour favoriser la meilleure qualité architecturale possible dans le nouveau projet: une étude détaillée des caractéristiques formelles du bâtiment original devrait être réalisée le plus tôt possible, afin de mieux connaître le bâtiment et d'en expliquer tous les vestiges ${ }^{13}$. Cette étape nous paraît essentielle à la réalisation d'un projet architectural de qualité.

\section{Esquisse d'un concept pour la reconstruction du Manège militaire}

Les esquisses qui suivent illustrent les éléments de base d'un concept architectural qui s'appuie sur les principes définis dans la première section. Les principes et les esquisses constituent des réflexions distinctes, bien qu'interdépendantes. Il existe de toute évidence une grande variété de solutions formelles pouvant répondre aux principes fondamentaux que nous venons d'énoncer: le défi se situe justement dans le développement d'une expression contemporaine compatible avec la restauration des parties conservées du bâtiment, et travaillant à la mise en valeur des idées fondamentales de l'architecte Taché.

\section{La façade nord}

Dans la façade principale (fig. $\mathbf{n}^{\circ} \mathbf{1 0}$, en haut) donnant sur le terrain de parade et la Grande-Allée, l'harmonie des matériaux, le rythme serré des éléments répétitifs et la présence des tours et pavillons doivent d'abord être pris en considération. Si on recherche une unité d'ensemble, le caractère sériel des éléments conservés (contreforts, fenêtres et lucarnes passantes) devrait suggérer un design aux caractéristiques complémentaires (répétition des lucarnes supérieures et des joints de la couverture de cuivre, aspect sériel de l'ornement du faîte). Moins pour restaurer que pour construire d'une manière cohérente avec les éléments conservés, l'esquisse cherche à retrouver le caractère original du bâtiment de Taché. 

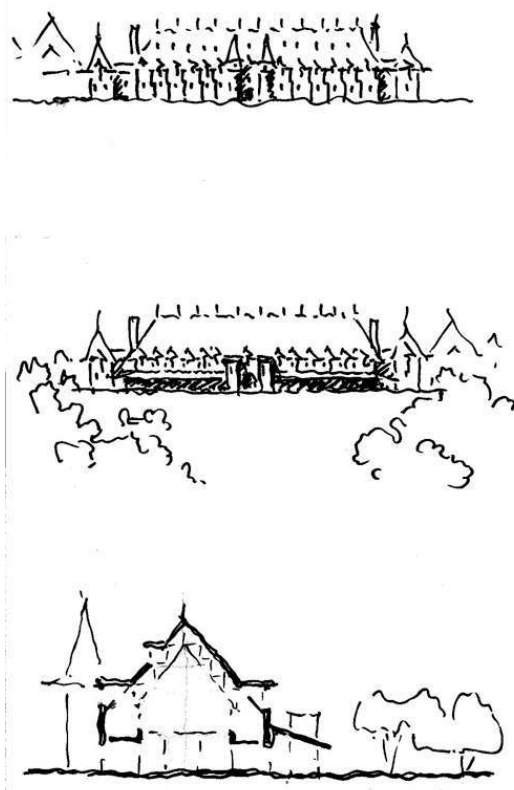

Esquisse d'un concept pour la reconstruction du Manège militaire, par Ayşe Orbay, 2009-2010 : façade nord (en haut), façade sud (au centre), et coupe (en bas).

(c) Ayşe Orbay, architecte.

\section{La façade sur les Plaines d'Abraham}

L'ancienne section en appentis est réinterprétée de manière à mieux dégager visuellement le bâtiment principal (voir fig. $\mathbf{n}^{\circ} \mathbf{1 0}$, au centre). D'un côté, elle loge un café et donne accès au Musée des Voltigeurs; de l'autre elle contient des espaces à bureaux et un petit centre d'information, où de la documentation sur les Plaines d'Abraham pourrait être rendue disponible.

La toiture de l'appentis est recouverte de cuivre, comme les autres toitures du bâtiment. Son mur extérieur, en revanche, est en verre avec une structure d'acier dont le rythme répond de manière allégée au bâtiment. Au centre, l'appentis s'arrête sur les deux tours d'escalier, pour laisser la grande porte du manège visible et accessible directement de l'extérieur, comme Taché le voulait initialement.

Cette réinterprétation de l'appentis permet de bien mettre en évidence le rythme des éléments composant le volume principal (contreforts, lucarnes). Le contraste entre les façades nord et sud correspond à la destination de chacune. Sur la Grande-Allée, l'aspect militaire original est conservé ; sur le parc, la façade devient plus ouverte et accueillante, tout en répondant à la monumentalité de la première. 


\section{Plan d'ensemble}

Le bâtiment principal loge une salle multifonctionnelle avec mezzanine agrandie; l'appentis qui longe l'édifice sur le côté sud est interrompu au centre ; les tours d'escalier au sud sont exprimées comme des volumes indépendants; un aménagement paysager relie le manège au parc des Plaines d'Abraham (fig. $\mathbf{n}^{\circ} \mathbf{1 1}$ ).

Afin de réaliser un aménagement paysager qui mette l'emphase sur la fonction d'accueil pour les promeneurs, le stationnement qui ceinture le Manège militaire est éliminé. Il reste les espaces de stationnement situés de part et d'autre de l'ensemble. Mais la verdure rejoint le bâtiment et l'entoure complètement.

Figure 11

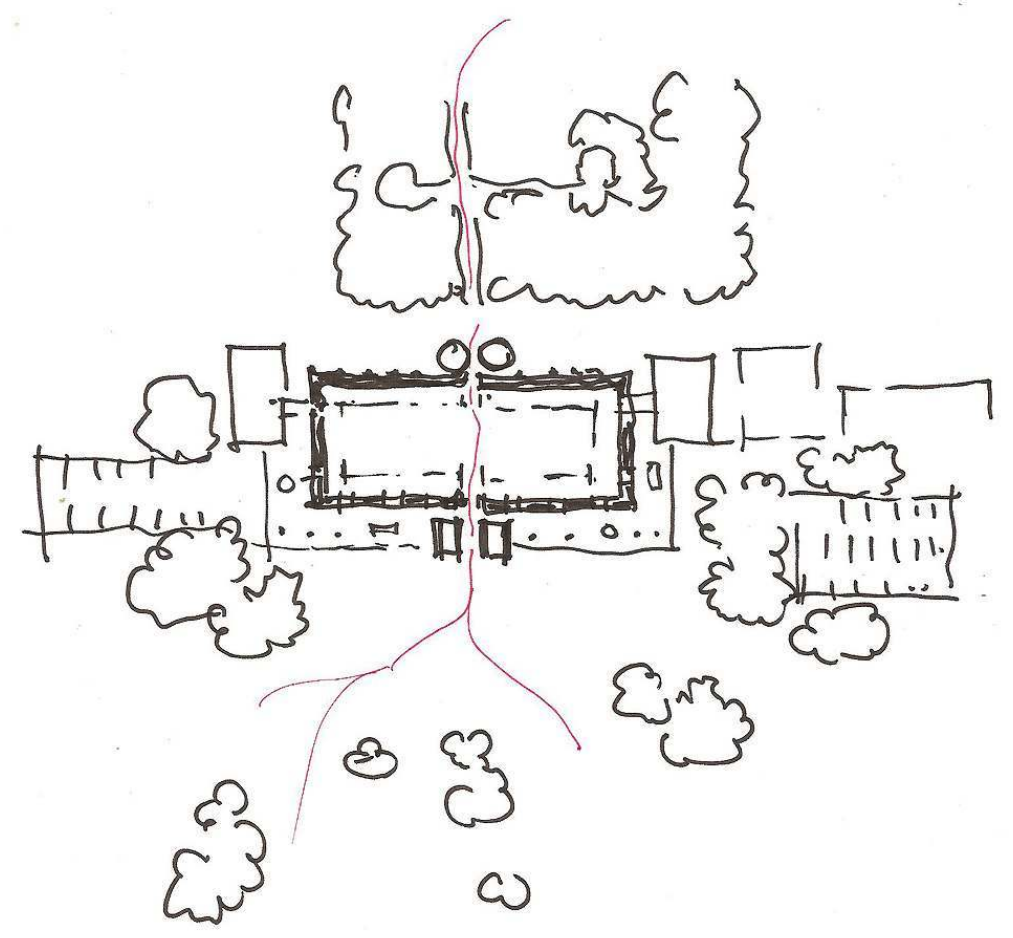

Esquisse d'un concept pour la reconstruction du Manège militaire, par Ayşe Orbay, 2009-2010 : plan d'ensemble.

(c) Ayşe Orbay, architecte.

\section{La forme du bâtiment vue en coupe}

Les coupes transversales montrent le Manège militaire avec sa grande toiture rétablie (voir fig. $\mathbf{n}^{\circ} \mathbf{1 0}$, en bas). À l'intérieur, la charpente reste ouverte au-dessus de la salle multifonctionnelle. La mezzanine est suffisamment grande pour accueillir des vitrines d'exposition destinées au Musée des Voltigeurs.

L'idée originale de transformer en mezzanine le surcroit de l'architecture renaissante constitue le point de départ fondamental de notre réflexion, car elle est au cœur des idées de Taché. Les lucarnes passantes sont aussi utilisées à leur plein potentiel. 
Sur le côté sud, la première coupe montre la structure en appentis, dont la toiture s'appuie sur le bâtiment principal au niveau de la mezzanine. La seconde explique la disposition des nouvelles tours d'escalier, qui donnent accès à la mezzanine par une passerelle, puisqu'elles sont placées à une certaine distance du bâtiment principal. De cette manière, les caractéristiques fondamentales du bâtiment sont bien mises en évidence, tant du côté nord que du côté sud.

\section{Conclusion}

Nous avons tenté de démontrer que, pour bien mettre en valeur le Manège militaire, sa reconstruction devrait s'appuyer sur les principes fondamentaux qui étaient à la base de l'architecture d'Eugène-Étienne Taché. Comme l'essentiel de la maçonnerie du bâtiment principal a été conservé, et que les parties secondaires ont été moins touchées par l'incendie, l'organisation spatiale de base de l'ensemble est parfaitement reconnaissable et doit être respectée. Dans la maçonnerie, les parties abîmées telles que les lucarnes passantes et les cheminées peuvent être rétablies, et par conséquent devraient l'être. La grande toiture devrait aussi être reconstruite d'une manière assez proche de sa forme originale, afin conserver la cohérence visuelle des différents volumes. Une attention particulière devrait être portée à la relation de complémentarité entre les matériaux extérieurs (cuivre et pierre) et à l'aspect sériel des éléments architecturaux.

L'intérieur, qui a été complètement détruit, appelle une réflexion plus théorique, centrée sur les qualités architecturales essentielles du Manège militaire original plutôt que sur la forme précise des éléments disparus. Ce sont les qualités spatiales, intimement liées aux proportions du périmètre de maçonnerie et à sa fenestration, qui devraient d'abord être mises en valeur, car celles-ci survivent très concrètement dans les vestiges conservés. Nous considérons l'étendue de la grande salle d'exercice originale comme un élément fondamental de cet intérieur. À ceci s'ajoute la position de la mezzanine vue en coupe, qui est un autre élément essentiel du bâtiment, notamment parce qu'elle constitue la clé de l'interprétation que Taché a faite de la Renaissance française. Par ailleurs, il est clair que ces qualités architecturales doivent être mises au service des besoins actuels, et que la forme précise des éléments détruits par l'incendie peut être modifiée.

Le projet d'architecture qui sera retenu devra donc réussir à maintenir un équilibre difficile entre le respect des formes originales et le design contemporain. Si nous ne favorisons pas la reconstitution systématique des éléments disparus, nous sommes néanmoins convaincus que les qualités architecturales des vestiges doivent être exploitées de la manière la plus directe possible. Et pour cela, les vestiges doivent d'abord être étudiés d'une manière approfondie. Si la reproduction nostalgique de l'ancien est une attitude ayant peu d'intérêt à nos yeux, l'utilisation de l'ancien comme simple décor accompagnant le nouveau est tout aussi problématique. Nous espérons vivement que la reconstruction du Manège militaire proposera une architecture de qualité, dont la forme montrera comment le présent est capable de se reconnaitre dans le passé. 


\section{NOTES}

1. - Marc Grignon est professeur d'histoire de l'architecture à l'Université Laval à Québec. Il est l'auteur de «Loing du Soleil. » Architectural Practice in Quebec City during the French Regime. New York: Peter Lang, 1997, et de plusieurs articles scientifiques sur l'architecture de Québec. Ayşe Orbay est architecte spécialisée en restauration et travaille à Istanbul. Elle a réalisé le Musée Erçumend Kalmik (1996) à Istanbul et elle vient de compléter la restauration de la Médersa Rabi (XVI ${ }^{\mathrm{e}}$ siècle), une composante du complexe de la Mosquée de Soliman le Magnifique.

2. - Les auteurs remercient Mme Aude Gendreau-Turmel qui, à titre d'auxiliaire de recherche, a beaucoup facilité la préparation de ce document.

3. - Une enquête a depuis permis d'identifier la cause de l'incendie : une lampe halogène placée temporairement pendant l'installation d'un système de gicleurs automatiques pour la protection de l'édifice contre le feu, était restée allumée par erreur pendant la nuit et serait tombée au sol. Voir BOIVIN, Matthieu. L'incendie du Manège militaire deux ans après, Le Soleil (Québec), 2 avril 2010, en ligne sur Cyberpresse : www.cyberpresse.ca [consulté le 12 juin 2010].

4. - BAILLARGEON, Jean. Rapport final - Consultation publique sur l'avenir du Manège militaire de la Grande-Allée à Québec, 29 septembre 2009. Internet: TPSGC, Manège militaire de la Grande-Allée à Québec: http://www.tpsgc-pwgsc.gc.ca/que/region/text/manegearmoury/rapport-report/index-fra.html [page consultée le 12 juin 2010].

5. - BUSSIÈRES, Ian. Manège militaire : pas avant 2016... si tout va bien, Le Soleil (Québec), 11 juin 2010, en ligne sur Cyberpresse : www.cyberpresse.ca [consulté le 12 juin 2010]. Voir aussi le site de TPSGC : http://www.tpsgc-pwgsc.gc.ca/que/region/text/manege-armoury.

6. - BUSSIÈRES, Ian. Manège militaire : pas avant 2016... si tout va bien, Le Soleil (Québec), 11 juin 2010, en ligne sur Cyberpresse : www.cyberpresse.ca [consulté le 12 juin 2010].

7. - «Son style précurseur de plusieurs autres bâtiments d'importance au Canada a d'ailleurs mené à sa désignation de 'lieu historique national du Canada' sur la recommandation de la Commission des lieux et des monuments historiques du Canada, ce qui inclut le champ de parade (ou place Georges-V), situé entre le bâtiment et la Grande-Allée côté Nord. L'édifice a aussi été 'classé' sur la recommandation du Bureau d'examen des édifices fédéraux du patrimoine, c'est-àdire qu'il a obtenu la plus haute désignation patrimoniale qui puisse être accordée à un édifice fédéral par le ministre de l'Environnement »: TPSGC. Manège militaire de la Grande-Allée de Québec, Internet: http://www.tpsgc-pwgsc.gc.ca/que/region/text/manege-armoury [page consultée le 21 juin 2010].

8. - Voir «Consultation publique / Paramètres établis» In : TPSGC, Manège militaire de la Grande-Allée de Québec, Internet: http://www.tpsgc-pwgsc.gc.ca/que/region/text/manegearmoury [page consultée le 26 mai 2009].

9. - Voir ANDROUET DU CERCEAU, Jacques. Les trois livres d'architecture: 1559, 1561, 1582, rééd. Ridgewood, N.,J., Gregg Press, 1965.

10. - Voir FOGELSON, R.M. America's Armories: Architecture, Society, and Public Order. Cambridge, Mass.: Harvard University Press, 1989.

11. - Le Chalet du Mont-Royal à Montréal, conçu par Aristide Beaugrand-Champagne, a été érigé en 1931-32. Été comme hiver, il sert d'abri aux promeneurs (incluant skieurs et raquetteurs), qui peuvent s'asseoir et se reposer, manger en famille la nourriture qu'ils ont apportée (piquenique), ou acheter des goûters légers à un petit comptoir. 
12. - Voir « Drill Hall, Quebec », dessins architecturaux, Fonds Taché, BaNQ, Centre d'archives de Québec.

13. - L'existence du Fonds Taché conservé au Centre d'archives de Québec (Bibliothèque et archives nationales du Québec) facilitera énormément ce travail.

\section{RÉSUMÉS}

Le Manège militaire de Québec (Eugène-Étienne Taché, 1887), détruit par un violent incendie en avril 2008, était un des édifices les plus remarquables de la "Vieille Capitale ", aux côtés de l'hôtel le Château Frontenac et du Parlement québécois. En vue d'une éventuelle reconstruction, le service des travaux publics canadiens a lancé un processus de consultation publique et a reçu les propositions et commentaires en mai 2009. Un mémoire soumis par les deux auteurs constitue la base de cet article qui fait le point, en première partie, sur les caractéristiques architecturales du bâtiment de Taché et tente de dégager des principes qui, à leur avis, devraient constituer les fondements du projet de reconstruction. En tenant compte de l'état des restes du bâtiment, les auteurs ne proposent pas la reconstitution de l'édifice original, mais plutôt une interprétation actuelle basée sur les principes architecturaux de Taché, avec une restauration partielle des éléments les mieux conservés, essentiellement l'extérieur. Dans la seconde partie, les dessins d'A. Orbay donnent les grandes lignes d'un projet qui pourrait répondre aux principes dégagés précédemment. Les auteurs suggèrent que le bâtiment devienne un point d'accueil pour les visiteurs du Parc des Champs de Bataille (ou Plaines d'Abraham), avec une grande salle multifonctionnelle ouverte aux promeneurs et pouvant accueillir des cérémonies officielles et des expositions - à la manière de l'édifice original. Les dessins ne constituent pas un véritable avant-projet, mais visent surtout à illustrer le potentiel des principes formulés dans la première partie.

The Grande-Allée Armoury in Quebec City (Eugène-Étienne Taché, 1887), destroyed by fire in April 2008, was one of the most remarkable historical buildings in the 'Old Capital', together with the hotel Château Frontenac and the Quebec Parliament. Considering the possibility of rebuilding the armoury, the Public Works service of the Canadian government launched a process of public consultation and received proposals and comments in May 2009. A memoir submitted by the two authors is the main source for this article which, in the first part, identifies the most important architectural features of Taché's design, and draws up principles that should, in their view, provide the basis of the reconstruction project. Taking into account the condition of the remains of the buildings, the authors are not proposing a reconstitution of the original design, but rather a contemporary interpretation based on Taché's architectural ideas, and a partial restoration of the elements that are best preserved, essentially the exterior of the building. In the second part of the article, drawings made by A. Orbay show the general appearance of a project that would conform to the principles established earlier. The authors propose that the building become a visitors' centre for the Battlefields Park (or Plains of Abraham), with a multipurpose space open to visitors and suitable for holding special events such as official ceremonies and exhibitions - in a manner akin to that of the original building. These drawings are not meant as the draft of a specific projet, they rather aim at showing the potential of the principles formulated in the first part of the article and complete them with illustrations. 
INDEX

Keywords : armoury, Eugène- Étienne Taché, historic monument, Plains of Abraham, public consultation, Quebec (city), reconstruction, restoration

Mots-clés : consultations publiques, Eugène-Étienne Taché, manège militaire, monuments historiques, Plaines d'Abraham, Québec (ville), reconstruction, restauration

\section{AUTEURS}

\section{MARC GRIGNON}

Département d'histoire/CÉLAT, Université Laval, Québec Marc.Grignon@hst.ulaval.ca AYSE ORBAY

architecte, Istanbul aorbay@ttmail.com 